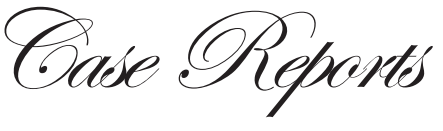

\section{Macronodular tuberculosis of the liver: a tumour masquerade}

\section{Introduction}

Liver involvement in tuberculosis, although common in both pulmonary and extrapulmonary tuberculosis, is usually clinically silent. ${ }^{1}$ Isolated macronodular hepatic tuberculosis is the rarest form of hepatic tuberculosis. ${ }^{1,2}$ Although the incidence of tuberculosis had initially decreased with the advent of effective chemotherapy, it is currently increasing especially among the elderly, the immunocompromised and those from the lower socioeconomic strata. ${ }^{3}$ Diagnosis of nodular hepatic tuberculosis is not straightforward, especially in the absence of pulmonary /miliary tuberculosis as this lesion is a tumour mimic on imaging. ${ }^{1,2}$ The diagnosis of macronodular tuberculosis is usually unsuspected and confused with primary or metastatic carcinoma and as in our case, it may necessitate a hepatic resection.

\section{Case report}

A 50-year-old female presented with complaints of upper abdominal pain for one month and low grade pyrexia for 10 days. She also complained of anorexia and significant weight loss. She had no comorbid illnesses and no past or family history of contact with tuberculosis. At presentation she was thin and anicteric. Her general and abdominal examination were unremarkable and her vitals were stable. Her complete hemogram was within normal limits, apart from a raised ESR of $40 \mathrm{~mm} / \mathrm{hr}$ and her liver function tests were within normal limits. The serum á-fetoprotein was $2.31 \mathrm{ng} / \mathrm{mL}$ (normal range 0-9.0 $\mathrm{ng} / \mathrm{mL}$ ) and carcinoembryonic antigen was $1.47 \mathrm{ng} / \mathrm{mL}$ (normal range $0-3.0 \mathrm{ng} / \mathrm{mL}$ ). Viral markers for hepatitis $\mathrm{B}$ and $\mathrm{C}$ were also negative. The chest $\mathrm{X}$-ray showed no obvious abnormalities. Ultrasonography showed a solid-appearing, confluent, irregular, hypoechoic mass lesion in segments VI and VII of the right hepatic lobe, measuring $7 \times 5 \mathrm{~cm}$, with evidence of flow within the lesion. Contrast-enhanced CT showed a $7 \times 4 \mathrm{~cm}$, mixed density lesion in segments VI and VII. Peripheral enhancement with central non-enhancing areas after intravenous injection of contrast in the arterial phase with persistent opacification in venous and delayed phases was noticed (Figure 1). Her esophagoduodenoscopy and colonoscopy were normal. Since the diagnosis was inconclusive, we performed a fine-needle aspiration cytology (FNAC) of the lesion which also gave inconclusive results. Therapeutic options were discussed with the patient and she was offered surgery. She underwent a diagnostic laparoscopy which revealed a cirrhotic liver, with left lobe atrophy and a compensatory right lobe hypertrophy. The space occupying lesion (SOL) was noted in segments VI and VII of the liver and was found adherent and infiltrating the right adrenal gland. The right kidney was free. Since the patient had cirrhosis and we made a provisional preoperative diagnosis of a malignant liver SOL. A wedge resection of the liver lesion was performed with a $2 \mathrm{~cm}$ three dimensional margin and an en-bloc right adrenalectomy (Figure 2). Ex-vivo aspirate of the specimen

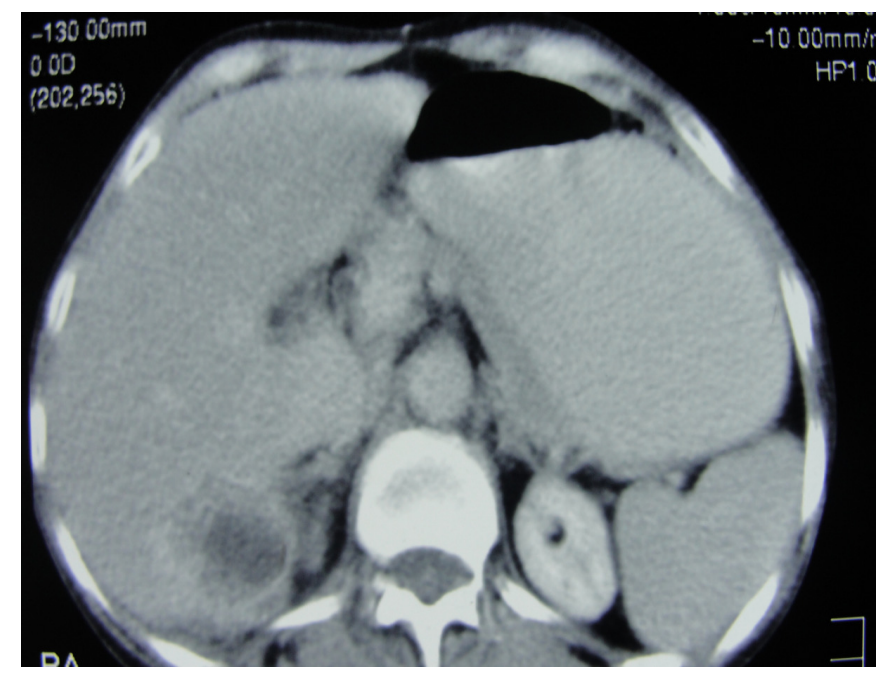

Figure 1: CECT showing a mixed density lesion in segments VI and VII of liver.

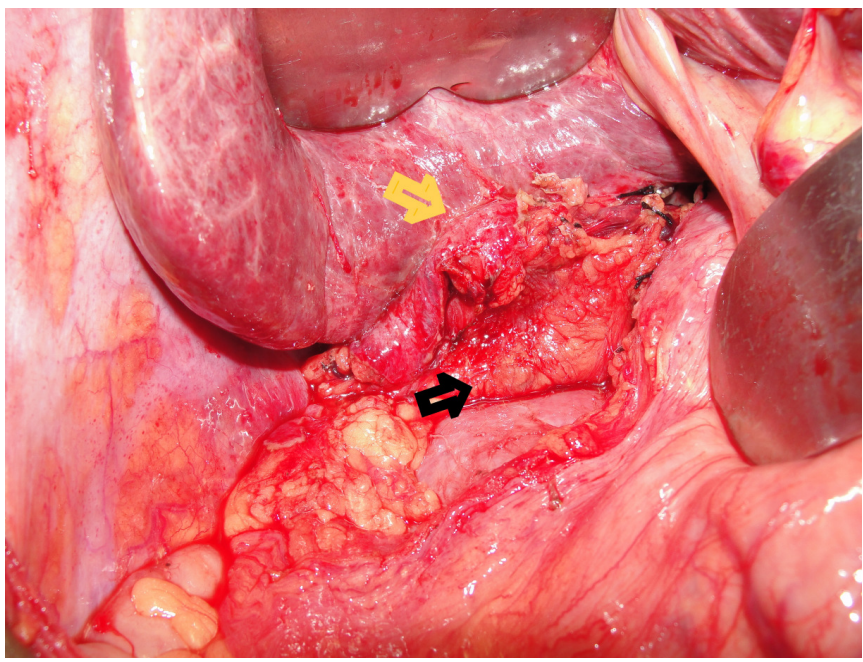

Figure 2: Intraoperative view showing the liver space occupying lesion (yellow arrow) being excised en-bloc with the right adrenal (black arrow). 
revealed acid-fast bacilli (AFB) on Ziehl Neelsen staining. Histopathology revealed multiple caseating granulomata bordered by multinucleate giant cells. The surrounding liver parenchyma showed lymphocytic infiltration within the portal tracts with occasional eosinophils. These findings were consistent with hepatic tuberculosis. Her postoperative period was uneventful and she was discharged on the $6^{\text {th }}$ postoperative day. She was started on quadruple anti-tuberculosis therapy and is asymptomatic on follow-up, tolerating her medication well.

\section{Discussion}

Liver is a rare site for tuberculosis. Tuberculosis of the liver has been classified into six different types namely liver involvement in miliary tuberculosis, pulmonary tuberculosis with liver involvement, primary liver tuberculosis, focal tuberculoma, tuberculous abscess and tuberculous cholangitis. ${ }^{4}$ Isolated giant solid macronodular tuberculosis (tuberculoma of liver) with no clinical extrapulmonary manifestations is the rarest form. ${ }^{1,2,4}$ Hepatic tuberculosis with mass lesions larger than $2 \mathrm{~mm}$ in diameter are referred to as macronodular or pseudotumoural tuberculosis. ${ }^{4,5}$

The presentation can be protean, with nonspecific abdominal pain, fever and weight loss being the most common symptoms. Clinical signs are nonspecific and hepatomegaly may be the only abdominal sign in this condition. ${ }^{2,5,6}$ Biochemical studies reveal no consistent findings, raised serum alkaline phosphatase levels have been the frequently noted. Tuberculous cholangitis may present with obstructive jaundice due to enlarged lymph nodes. Others findings may include anaemia, hypoalbuminaemia, and hyponatraemia. ${ }^{2.5,6}$ On the basis of imaging alone, these lesions are indistinguishable from other neoplastic lesions of the liver. Imaging techniques (ultrasonography, CT and MRI) are useful in diagnosing tuberculoma or tubercular abscess. On CECT, they appear as non-enhancing, central, low density lesions due to the caseation necrosis with a slightly enhancing peripheral rim due to the surrounding granulation tissue. When multiple, these lesions typically show varying densities due to the coexistence of different stages of granuloma formation, liquefaction, necrosis, fibrosis and calcification. ${ }^{4,7}$ On MRI these lesions appear as a hypointense nodule with a hypointense rim on T1weighted imaging and a hypointense, isointense or hyperintense lesion with a less intense rim on T2-weighted imaging with peripheral enhancement. ${ }^{4,7}$ Aspirates from the lesion for AFB staining have shown a sensitivity of $0-45 \%$, while cultures are positive in only 10 to $60 \%$ cases. With a sensitivity of $57 \%$ the results of polymerase chain reaction have also been disappointing. ${ }^{3,8}$ Pathological examination is necessary for diagnosis. ${ }^{1,2,5,6}$ Histologically, the presence of a caseating granuloma is diagnostic of hepatic tuberculosis. ${ }^{2,5}$ Quadruple therapy (isoniazid, rifampin, pyrazinamide and ethambutol) is recommended because of the increasing incidence of drug-resistant tuberculosis. A one year course of therapy is recommended. ${ }^{2,3,5,6}$ With early diagnosis and effective treatment, the prognosis of hepatic tuberculosis is usually good. ${ }^{2,6}$

\section{Conclusion}

Tuberculosis presenting as an isolated liver tumour, without active pulmonary or miliary tuberculosis or other evidence of tuberculosis is distinctly rare. These lesions are easily confused with more sinister liver neoplasms. Hence the need for increased awareness of these tumour mimics, particularly in endemic areas, to avert unnecessary morbidity from surgery.

ASHWIN RAMMOHAN

JESWANTH SATHYANESAN RAVICHANDRAN PALANIAPPAN G MANOHARAN

Correspondence: Dr. Ashwin Rammohan Institute of Surgical Gastroenterology and Liver Transplantation, Centre for GI Bleed,

Division of HPB Diseases, Stanley Medical College Hospital, Old Jail Road, Chennai, India Email: ashwinrammohan@gmail.com

\section{References}

1. Vimalraj V, Jyotibasu D, Rajendran S, Ravichandran P, Jeswanth S, Balachandar TG, et al. Macronodular hepatic tuberculosis necessitating hepatic resection: a diagnostic conundrum. Can J Surg. 2007;50:E7-8.

2. Desai CS, Josh AG, Abraham P, Desai DC, Deshpande RB, Bhaduri A, et al. Hepatic tuberculosis in absence of disseminated abdominal tuberculosis. Ann Hepatol. 2006;5:41-3.

3. Hsieh TC, Wu YC, Hsu CN, Yang CF, Chiang IP, Hsieh CY, et al. Hepatic macronodular tuberculoma mimics liver metastasis in a patient with locoregional advanced tongue cancer. J Clin Oncol. 2011;29:e641-3.

4. Levine C. Primary macronodular hepatic tuberculosis: US and CT appearances. Gastrointest Radiol. 1990;15:307-9.

5. Mert A, Ozaras R, Tabak F, Ozturk R, Bilir M. Localized hepatic tuberculosis. Eur J Intern Med. 2003;14:511-2. 
6. Huang WT, Wang CC, Chen WJ, Cheng YF, Eng HL. The nodular form of hepatic tuberculosis: a review with five additional new cases. J Clin Pathol. 2003;56:835-9.

7. Yu RS, Zhang SZ, Wu JJ, Li RF. Imaging diagnosis of 12 patients with hepatic tuberculosis. World $J$ Gastroenterol. 2004;10:1639-42.

8. Diaz ML, Herrera T, Lopez-Vidal Y, Calva JJ, Hernandez R, Palacios GR, et al. Polymerase chain reaction for the detection of Mycobacterium tuberculosis DNA in tissue and assessment of its utility in the diagnosis of hepatic granulomas. J Lab Clin Med. 1996;127:359-63.

\section{Lymphoepithelioma-like carcinoma: an unusual gall bladder tumour}

\section{Introduction}

Lymphoepithelioma-like carcinoma (LLC) of the biliary tract is a relatively recently recognized entity. LLC involving the gall bladder (LLC-GB) has been described only once in the literature. ${ }^{1}$

\section{Case report}

A 64-year-old lady presented with right upper abdominal pain of three months duration. Clinical examination was normal. CT scan (Figure 1) revealed a $2.4 \times 2.0 \times 3.1 \mathrm{~cm}$ heterogeneously enhancing soft tissue lesion arising in relation to the gall bladder neck. The common bile duct was dilated $(10 \mathrm{~mm})$ till its lower end with no evidence of obstruction at the ampulla. Subcentimetric lymph nodes were present in the hepatoduodenal ligament. A provisional diagnosis of carcinoma gall bladder was made and the patient was taken up for surgery. At surgery, there was a $3 \mathrm{~cm}$ mass arising from the gallbladder neck and confined to the gall bladder. There were no peritoneal or liver metastases. Extended cholecystectomy was performed. Cystic duct margin was free of tumor on frozen section analysis. The postoperative course was uneventful and the patient was discharged in satisfactory condition. Histopathology of the resected specimen showed surface epithelial ulceration and infiltration by a tumor composed of syncytial sheets and islands of atypical polygonal cells intermixed and surrounded by

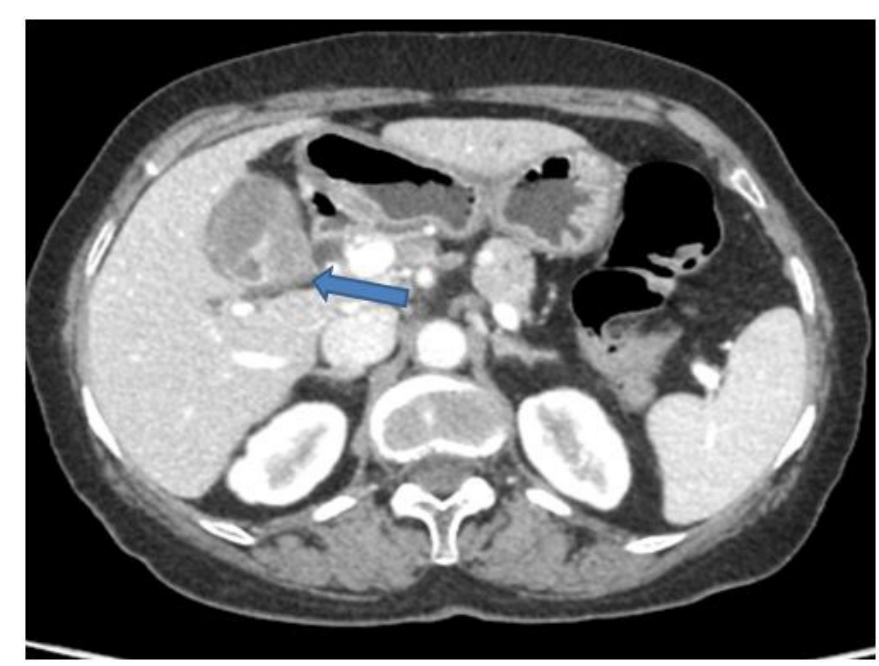

Figure 1: CECT showing mass in the neck of gall bladder (arrow)

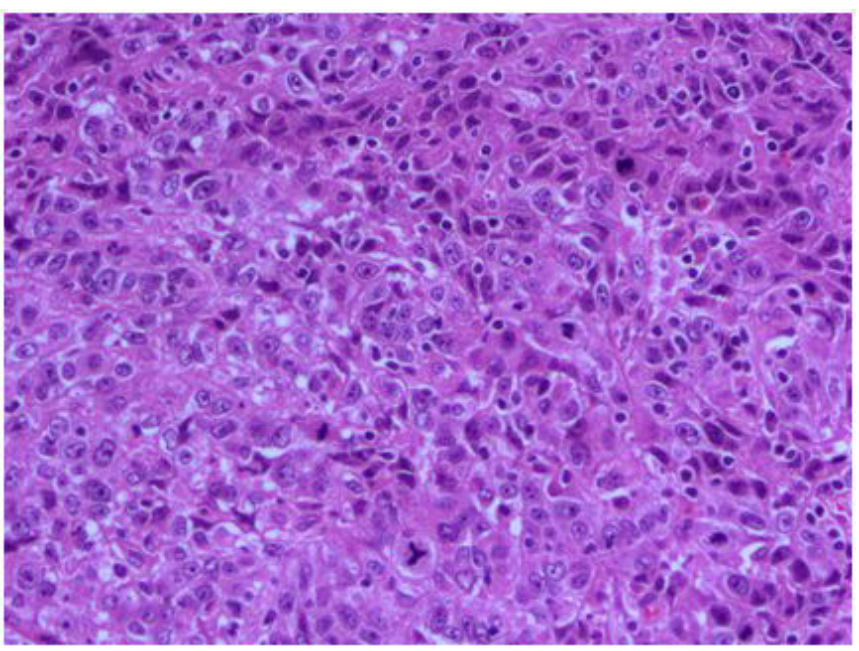

Figure 2: Tumor histopathology showing syncytial sheets and islands of atypical polygonal cells intermixed and surrounded by benign lymphoid cells (haematoxylin and eosin, 100x)

lymphoid cells (Figure 2). The tumor had infiltrated the mucosa, muscularis propria and had reached up to the subserosa. Perineural invasion was seen but there was no lymphovascular invasion. Cystic duct margin, liver transection margin and all resected lymph nodes were free of tumour. Tumour cells showed positive cytoplasmic staining with pancytokeratin and were for negative for Epstein-Barr virus on immunohistochemistry. Patient has been followed up for 18 months after surgery with no evidence of recurrence.

\section{Discussion}

Schmincke in 1921 described a distinct type of undifferentiated nasopharyngeal carcinoma where tumor cells grow in a diffuse manner mimicking a malignant lymphoma. ${ }^{2}$ This tumor has been designated as lymphoepithelial carcinoma or undifferentiated 